\title{
Felicidade, bem-estar subjetivo e variáveis sociodemográficas, em grupos de estudantes universitánios
}

\author{
JośAugustoDdaCdea - UnivesidadedeUbelânda, MinasGeais, Brasil \\ JoǽEduardbFenera Lqpes- UnivesidadedeUbelânda, Minas Gerais, Brasil \\ Marilia Fereira Deda Cdea - UnivesidadedeUbelânda, MinasGerais, Brasil
}

\begin{abstract}
Resumo
Este estudo foi conduzido junto a 388 estudantes universitários, utilizando um conjunto de escalas para medida de variáveis associadas à felicidade, bem-estar subjetivo, comportamento social e algumas questões sobre dados biográficos. Os resultados mostraram que as escalas apresentam, em geral, altos índices de confiabilidade, resultados médios superiores aos esperados nas medidas de bem-estar subjetivo e coeficiente de correlação múltipla de 0,79 na predição da felicidade total. Ao se testarem as diferenças entre as médias dos diversos grupos, constituídos por suas características biográficas, verificou-se que as mulheres, os mais jovens, os casados ou namorados, os religiosos, os que estudaram em escolas privadas no ensino fundamental e públicas no ensino superior, que conhecem outros idiomas, com renda familiar mais alta, com altos níveis de escolaridade dos pais, com mais altos níveis socioeconômicos mostram-se significativamente mais populares, menos solitários, mais satisfeitos com a vida e mais felizes.
\end{abstract}

Palamasdhave Felicidade; Bem-estar Subjetivo; Estudantes universitários; Suporte social.

\section{Happiness, subjective well-being and socio-demographic variables in groups of college students}

\begin{abstract}
This study was developed with 388 college students using a set of scales to measure variables associated to happiness, subjective well-being, social behavior and some questions on demographic data. The results showed that the scales usually present high levels of reliability, average results superior to those expected in the subjective well-being measures and multiple correlation coefficient of 0.79 on the prediction of total happiness. When testing the differences among the several groups, it was verified that women, young people, those married or dating, those who had a religion, studied the first grade in a private school or the college in a public institution, those who know other languages with higher family income, having parents with high level of education or social-economic status, showed to be significantly more popular, less solitaires, more satisfied with their lives and happier.
\end{abstract}

Kegnards Happiness; Subjective Well-being; College students; Social support.

\section{Felicidad, bienestar subjetivo y variables sociodemográficas, en gnupos de estudiantes universitarios}

\begin{abstract}
Resumen
Este estudio fue conducido con 388 estudiantes universitarios, utilizando un conjunto de escalas para medida de variables asociadas a la felicidad, bienestar subjetivo, comportamiento social y algunas cuestiones sobre datos biográficos. Los resultados mostraron que las escalas presentaron, en general, altos índices de confiabilidad, resultados medios superiores a los esperados en las medidas de bienestar subjetivo y coeficiente de correlación múltiple de 0,79 en la predicción de la felicidad total. En las pruebas de las diferencias entre los promedios de los diversos grupos, constituidos por sus características biográficas, se verificó que las mujeres, los más jóvenes, los casados o novios, los religiosos, los que estudiaron en escuelas privadas en la enseñanza básica y públicas en la enseñanza superior, que conocen otros idiomas, con renta familiar más alta, con altos niveles de escolaridad de los padres, con más altos niveles socioeconómicos se mostraron significativamente más populares, menos solitarios, más satisfechos con la vida y más felices.

Palabrasdave Felicidad; Bienestar subjetivo; Estudiantes universitarios; Soporte social.
\end{abstract}

Diversos indicadores podem sustentar a afirmação de que a Psicologia Positiva já se constitui em uma nova disciplina na área das ciências humanas e sociais. Eles incluem a publicação de manuais específicos sobre os temas por ela cobertos (Snyder \& Lopez, 2009), de obras de revisão (Diener, 2009), revistas especializadas, a ocorrência de congressos específicos da área, a existência de grupos de pesquisa dedicados a estudos em diversas partes do mundo, 0 enorme interesse e cobertura da mídia para os temas particulares, a criação de associações internacionais, entre outros (Linley, Joseph, Harrington \& Wood, 2006).
O domínio da Psicologia Positiva caracteriza-se pelo estudo de "[...] experiências subjetivas valiosas envolvendo o bem-estar, contentamento, satisfação (no passado), desejo e otimismo (para o futuro), deleite e felicidade (no presente)" (Seligman \& Csikszentmihalyi, 2000, p. 5). Este ramo do conhecimento dedica-se a estudar temas relacionados à felicidade, gratidão, bemestar subjetivo, satisfação com a vida, otimismo, esperança, virtudes, sabedoria, talento, fortalezas, resiliência, espiritualidade, perdão, tolerância, civilidade, responsabilidades, entre outros.

Melhor esclarecimento e diferenciação dos termos citados fogem ao objeto deste trabalho. Entretanto, como felicidade e bem-estar subjetivo serão justamente 
abordados ao Iongo do texto, seria conveniente esclarecer que Diener e Suh (2000) entendem genericamente que bem-estar subjetivo envolve as avaliações que as pessoas fazem de suas vidas, em seus diferentes aspectos, e que Seligman (2004) considera a felicidade, entre outros significados, como sentimento e cognição referente à comparação entre afetos positivos e afetos negativos experimentados pelo indivíduo.

No Brasil, do mesmo modo que em muitas partes do mundo, essa área, ainda que nova, apresenta publicações de diversos artigos, livros, dissertações e teses, realização de conferências, congressos, além dos citados neste trabalho, que já foram dedicados a alguns desses temas destacados anteriormente. Verifica-se que as publicações apresentam revisões (Ferraz, Tavares \& Zilberman, 2007; Passareli \& Silva, 2007), conceitos fundamentais (Giacomoni, 2004; Costa \& Pereira, 2007), desenvolvem e/ ou adaptam instrumentos para medidas das variáveis em estudo (Albuquerque \& Tróccoli, 2004), procedem a levantamentos e determinam parâmetros básicos das diversas variáveis para distintas amostras, replicam estudos conduzidos no exterior e mesmo testam algumas hipóteses já conhecidas e outras recém-formuladas (Albuquerque, Noriega, Coelho, Neves \& Martins, 2006; Albuquerque, Noriega, Martins \& Neves, 2008; Corbi \& Menezes-Filho, 2006; Rodrigues \& Pereira, 2007; Rodrigues \& Silva, 2010).

Os estudos inicialmente conduzidos nesta área procuraram verificar o nível de bem-estar de diferentes amostras, encontrando que os sujeitos brasileiros tendem a se posicionar em torno da média ou mediana, ou mesmo em posição superior, em relação a sujeitos de outros países, quando se consideram os mais variados aspectos da felicidade e do bem-estar subjetivo, mesmo quando se controlam os efeitos relacionados aos fatores econômicos (Dela Coleta \& D ela Coleta, 1997; D ela Coleta, D ela Coleta \& Diener, 1996; Diener, 2000; Diener, Diener \& Diener, 1995; Inglehart \& Klingemann, 2000; Schwartz \& Melech, 2000; Veenhoven, 2000).

Dela Coleta e Dela Coleta (2006), ao estudarem felicidade e bem-estar subjetivo em uma amostra de 252 estudantes universitários, entre muitos achados, encontraram resultados superiores à média, indicando sentimentos positivos de felicidade, satisfação geral e com diferentes aspectos da vida, gratidão, plenitude, deleite (flow), auto-avaliação do rendimento escolar e expectativas futuras, tanto do ponto de vista pessoal quanto profissional. Mostraram também que os sujeitos com níveis mais altos de felicidade, satisfação com a vida, bem-estar subjetivo apresentam mais elevadas autoavaliações como estudantes e condutas escolares mais apropriadas, tendo em vista 0 aproveitamento acadêmico .

Os mesmos autores (D ela Coleta \& D ela Coleta, 2007), trabalhando com 230 professores universitários, encontraram níveis superiores de sentimentos de felicidade, satisfação com a vida e com seus diferentes aspectos, sentimentos de gratidão, de deleite (flow, semelhantemente ao que ocorreu com grupos de estudantes universitários. 0 estudo mostrou também que os professores com maiores níveis de titulação, maior renda pessoal e familiar mostram-se mais felizes do que o grupo com níveis mais baixos dessas variáveis.

Entretanto, o mais importante achado relatado pelos autores diz respeito ao fato dos sujeitos com mais altos sentimentos de felicidade, gratidão e satisfação com a vida atribuírem mais esses estados a causas internas, pessoais, do que os sujeitos com níveis mais baixos, que preferem apontar as causas externas para explicar esses sentimentos.

$\mathrm{Na}$ literatura encontram-se inúmeros estudos, principalmente de natureza transcultural, que indicam diferenças importantes nas variáveis associadas ao bem-estar subjetivo, satisfação e felicidade, quando se consideram características biográficas, sociais e psicológicas dos sujeitos. Assim, Wilson (1967, p. 294), depois de diversos estudos, conclui que o sexo não tem influência na felicidade, chegando a afirmar que "[...] indivíduos felizes são de ambos os sexos". Lucas e Gohm (2000), contrariamente, concluíram que as mulheres tendem a experimentar mais afetos não prazerosos que os homens, mesma conclusão a que chegaram Diener, Suh, Lucas e Smith (1999), afirmando que os homens tendem a ser um pouco mais felizes que as mulheres, e que estas relatam mais afetos negativos, fato observado em quase todas as culturas. No Brasil, Guedea e cols. (2006) encontraram, em amostras de idosos, que as mulheres experimentam maiores níveis de satisfação com a vida, contrariamente a Gonçalves e Kapczinski (2008), que mostraram ser as mulheres menos satisfeitas com a vida e a Chaves e cols. (2002, citado por Chaves \& Fonseca, 2006), para quem amostras de homens de João Pessoa-PB experimentavam maiores níveis de bem-estar.

A revisão elaborada por Wilson (1967) mostrou que a idade está negativamente relacionada à felicidade, de modo que os jovens declaram experimentar níveis mais elevados dessa variável.

$\mathrm{Na}$ mesma direção, Lucas e Gohm (2000) afirmam que, em $90 \%$ das nações envolvidas em seus estudos, os sujeitos mostraram decréscimo dos afetos prazerosos com 0 acréscimo da idade. Contrariamente, no Brasil, Otta e Fiquer (2004) demonstraram que os idosos julgam-se mais satisfeitos com a vida, 
explicando que as emoções são melhor reguladas com 0 avanço da idade, fator que contribui para 0 sentimento de bem-estar mais positivo, o mesmo tendo sido encontrado por $\mathrm{G}$ onçalves e Kapczinski (2008).

Este tema ainda merece ser melhor explorado e tais diferenças entre os resultados dos estudos podem ser explicadas, em parte, pelo processo e escala de medida utilizados pelos pesquisadores em diferentes períodos, amostras e nacionalidades, ou mesmo, como preferem alguns, que a satisfação com a vida obedeceria uma distribuição em $U$, quando se considerasse a idade de alguns sujeitos.

Particularmente, ao buscar correlatos dos estados de satisfação, bem-estar subjetivo e felicidade com as condições econômico-financeiras, Diener e Oishi (2000) encontraram evidências que a prosperidade material e o crescimento econômico são desejos de muitas pessoas em diversas partes do mundo. Analisando resultados de vários estudos, em dezenas de países, conduzidos por eles e por outros pesquisadores, encontraram correlações importantes entre crescimento no ganho financeiro e bem-estar subjetivo, maiores entre amostras de nações pobres, concluindo que em diferentes sociedades o ganho econômico-financeiro determina o bem-estar subjetivo. Nesse sentido, afirmam que as pessoas tendem a ser mais felizes na medida em que conseguem as coisas que desejam, em um modelo que privilegia as necessidades objetivas. Correlações positivas e significativas foram também obtidas no Brasil, nos estudos de Corbi e Menezes-Filho (2006) e de Oliveira e cols. (2009) entre felicidade e renda.

Como forma de dar prosseguimento a esses avanços, confirmar os parâmetros básicos de algumas variáveis, estudar as correlações entre elas, testar algumas hipóteses, determinar a relação entre variáveis biográficas, socioeconômicas, e sentimentos de felicidade, gratidão e satisfação com a vida, desenvolveu-se o presente estudo, com universitários brasileiros.

\section{Amostra}

\section{Método}

Participaram deste estudo 388 estudantes, de todos os períodos, de diferentes cursos, de 11 instituições de educação superior, públicas e privadas, dos estados de Minas Gerais e Mato Grosso do Sul, com idade variando entre 18 e 40 anos, sendo 160 do sexo masculino e 228 do sexo feminino, caracterizando, dessa forma, uma amostra voluntária e não aleatória.

\section{Instrumentos}

Para a coleta de dados junto aos sujeitos deste estudo foi disponibilizado, em uma página acessada pela da internet, um conjunto de instrumentos contendo:

a) um inventário de dados biográficos, relativos aos dados pessoais, socioeconômicos e de escolarização do sujeito e de seus pais;

Em seguida, eram apresentados diversos conjuntos de instrumentos, todos em formato Liket, sendo os primeiros destinados à medida de diversas variáveis associadas à felicidade e ao bem-estar subjetivo, incluindo:

a) escala para medida dos sentimentos de satisfação geral com a vida (SATGERAL), com cinco itens;

b) escala para medida de satisfação com diferentes aspectos da vida (SATASPEC), com 26 itens;

C) escala para medida dos sentimentos de gratidão (GRAT), com seis itens;

d) escala para medida da felicidade comparativamente com outros (FELCOM), com quatro itens, as quatro escalas com sete opções de resposta (1 a 7);

e) escala para medida do nível geral de felicidade (FELICIDADE), com apenas um item, com onze alternativas para resposta (1 a 11);

f) escala para medida do sentimento de felicidade, comparativamente no tempo e aos outros (FELTEM), com oito itens, com cinco opções de resposta (1 a 5);

Todas essas escalas anteriormente descritas foram retiradas e/ ou adaptadas de estudos realizados por Dela Coleta e Dela Coleta $(2006,2007)$, Dela Coleta, Dela Coleta e Diener (1996), Diener, Diener e Diener (1995), Diener e Suh (2000), Seligman (2004) e apresentaram, nos estudos originais, bons índices de confiabilidade ( $\alpha$ entre 0,73 e 0,86 ) e de validade fatorial, indicando, em todas as análises, a presença de um único fator correspondente ao construto que se pretendia medir.

0 conjunto de instrumentos incluía também escalas para medida da satisfação com o suporte social e suas quatro subescalas, propostas por Ribeiro (1999), e quatro outras criadas para este estudo. 0 suporte social, tal como definido por Ribeiro (1999), "[...] é a existência ou a disponibilidade de pessoas em que se pode confiar, pessoas que nos mostram que se preocupam conosco, nos valorizam e gostam de nós" (p. 547).

a) escala para medida da satisfação com 0 suporte social em geral (SUPORSOC), com 15 itens e sete opções de resposta (1 a 7), compreendendo a satisfação com amizades (SATAMIZ, 5 itens e alfa de Cronbach do estudo original igual a 0,83 ), satisfação com aspectos 
relativos à intimidade das amizades (SA'I'IN' ', 4 itens e $\alpha=0,74)$, satisfação com 0 relacionamento familiar (SATFAM, 3 itens e $\alpha=0,74$ ) e satisfação com as atividades sociais (SATSOC, 3 itens e alfa $=0,64$ ), todas retiradas de Ribeiro (1999). A escala total apresenta alfa de 0,85.

i) escala, com item único, para medida do nível percebido de popularidade do sujeito (POPUL);

j) escala, com item único, para medida do nível percebido de não-solidão pelo sujeito (SOLID), apresentando ambas dez níveis de resposta (1 a 10);

Por último, dois itens foram propostos para a medida da percepção do nível sócio-econômico e do nível de justiça percebida deste nível:

a) escala, com um único item, para medida da percepção da posição do sujeito no continuo socioeconômico (ECONOM);

b) escala, com um único item, para medida da percepção da justiça desta posição socioeconômica, quando comparada a de seu melhor amigo (ECOJUST), ambas com dez níveis de resposta (1 a 10).

Como forma de elevar os níveis de confiança nas respostas dos sujeitos aos instrumentos foi introduzida uma "escala de mentira", com dois itens, aos quais os sujeitos deveriam, dada a obviedade das proposições, responder negativamente. Alguns poucos casos de respostas concordando com qualquer uma das duas proposições implicaram na eliminação dos sujeitos da amostra.

Ainda, dada a extensão dos instrumentos empregados neste estudo, na parte intermediária dos inventários foram incluídos dois itens distrativos, um que solicitava a indicação do número de bairros importantes existentes e outro que pedia uma estimativa do número de habitantes em sua cidade.

\section{Procedimentos}

Uma vez completadas as informações necessárias para esclarecimento dos sujeitos (Termo de Consentimento), as instruções para resposta, 0 inventario de dados pessoais e profissionais e as escalas para medida das diferentes variáveis constantes deste estudo, os autores fizeram contato com diversos coordenadores e professores, solicitando-lhes que pedissem a seus alunos que procurassem a referida página e, caso se sentissem suficientemente esclarecidos e estivessem de acordo com as tarefas exigidas e com os objetivos do estudo, respondessem aos instrumentos.

Após um período de dois meses de exposição da página, os dados recolhidos foram submetidos a diversos tratamentos estatísticos, valendo-se de procedimentos contidos no SPSS (Statistical Package for the Social Sciences) versão 17.0, lembrando que maiores escores em cada uma das escalas significam maiores níveis de presenças das variáveis.

\section{Resultados}

Inicialmente foram estudados os índices de confiabilidade das escalas utilizadas neste estudo com 0 intuito de verificar e confirmar dados obtidos por outros pesquisadores (Dela Coleta \& Dela Coleta, 2006; Ribeiro, 1999).

Os resultados indicam que as escalas empregadas para medida das diversas variáveis associadas à felicidade, gratidão e satisfação com diversos aspectos da vida apresentam coeficientes de confiabilidade $(\alpha$ de Crombach) variando entre 0,78 e 0,91, coeficientes de correlação item total entre 0,38 e 0,54, semelhantemente aos dados obtidos por D ela Coleta e D ela Coleta $(2006,2007)$ e Ribeiro (1999).

$\mathrm{Na}$ Tabela 1 podem ser observados os coeficientes de confiabilidade das escalas, as médias obtidas, as médias esperadas, caso as respostas fossem dadas ao acaso, e os desvios padrão das respostas. Os valores indicam médias obtidas significativamente superiores àquelas esperadas em todas as variáveis, exceção à satisfação com as atividades sociais das quais participa o sujeito, em que a média obtida é significativamente inferior à média esperada.

Tabela 1. Coeficientes de confiabilidade, médias esperadas e obtidas, resultados da comparação entre as médias, e desvios padrão dos escores das escalas utilizadas neste estudo ( $\mathrm{N}=388)$

\begin{tabular}{lcccrrr}
\hline Escala & No de itens & $\alpha$ de Cronbach & Média esperada & Média obtida & D esvio padrão & $t$ \\
\hline FELICIDADE & 1 & - & 6,00 & 7,73 & 2,40 & $14,19^{* *}$ \\
FELCOM & 4 & 0,83 & 22,00 & 27,99 & 6,92 & $17,05^{* *}$ \\
FELTEM & 8 & 0,84 & 24,00 & 29,97 & 4,65 & $25,28^{* *}$ \\
SATGERAL & 5 & 0,81 & 20,00 & 22,24 & 6,27 & $7,03^{* *}$ \\
SATASPEC & 26 & 0,91 & 104,00 & 136,64 & 20,50 & $31,36^{* *}$ \\
GRAT & 6 & 0,78 & 24,00 & 34,96 & 5,58 & $36,68^{* *}$ \\
SATAMIZ & 5 & 0,78 & 20,00 & 22,97 & 5,93 & $9,87^{* *}$ \\
SATFAM & 3 & 0,79 & 12,00 & 14,41 & 3,98 & $11,93^{* *}$ \\
\hline ** $<0,01$ & & & & &
\end{tabular}


Tabela 1. Coeficientes de confiabilidade, médias esperadas e obtidas, resultados da comparação entre as médias, e desvios padrão dos escores das escalas utilizadas neste estudo ( $\mathrm{N}=388)$

\begin{tabular}{lcccrrr}
\hline Escala & No de itens & $\alpha$ de Cronbach & Média esperada & Média obtida & D esvio padrão & t \\
\hline SATINT & 4 & 0,78 & 16,00 & 18,26 & 5,54 & $8,03^{* *}$ \\
SATSOC & 3 & 0,78 & 12,00 & 9,73 & 4,01 & $-11,15^{* *}$ \\
SUPORSOC & 15 & 0,83 & 60,00 & 65,36 & 13,87 & $7,61^{* *}$ \\
POPUL & 1 & - & 5,50 & 6,22 & 1,85 & $7,66^{* *}$ \\
SOLID & 1 & - & 5,50 & 6,76 & 2,62 & $9,44^{* *}$ \\
ECOJUST & 1 & - & 5,50 & 5,91 & 2,70 & $2,99^{* *}$ \\
ECONON & 1 & - & 5,50 & 4,96 & 1,62 & $6,57 * *$ \\
\hline
\end{tabular}

**p $<0,01$

Quando se analisam as médias das respostas relativas à satisfação dos sujeitos com cada um dos 26 aspectos de suas vidas, incluídos neste estudo, pode-se observar (Figura 1) que todas as médias apresentam-se superiores à média esperada $(\bar{X}=4,00)$, caso as respostas fossem dadas ao acaso. Comparando-se as médias da satisfação com cada um dos 26 aspectos da vida com a média geral das avaliações que os sujeitos fizeram da satisfação com estes diferentes aspectos de suas vidas ( $\bar{X}=5,26)$ pode-se verificar que eles estão muito mais satisfeitos com seus princípios morais, família, amigos, saúde, educação, liberdade, instrução, moradia e muito menos satisfeitos com finanças, emprego, amor, profissão, oportunidades e o país como um todo. Estes dados, em muitos aspectos, também puderam ser observados nas amostras de professores examinados por D ela Coleta e Dela Coleta (2007).

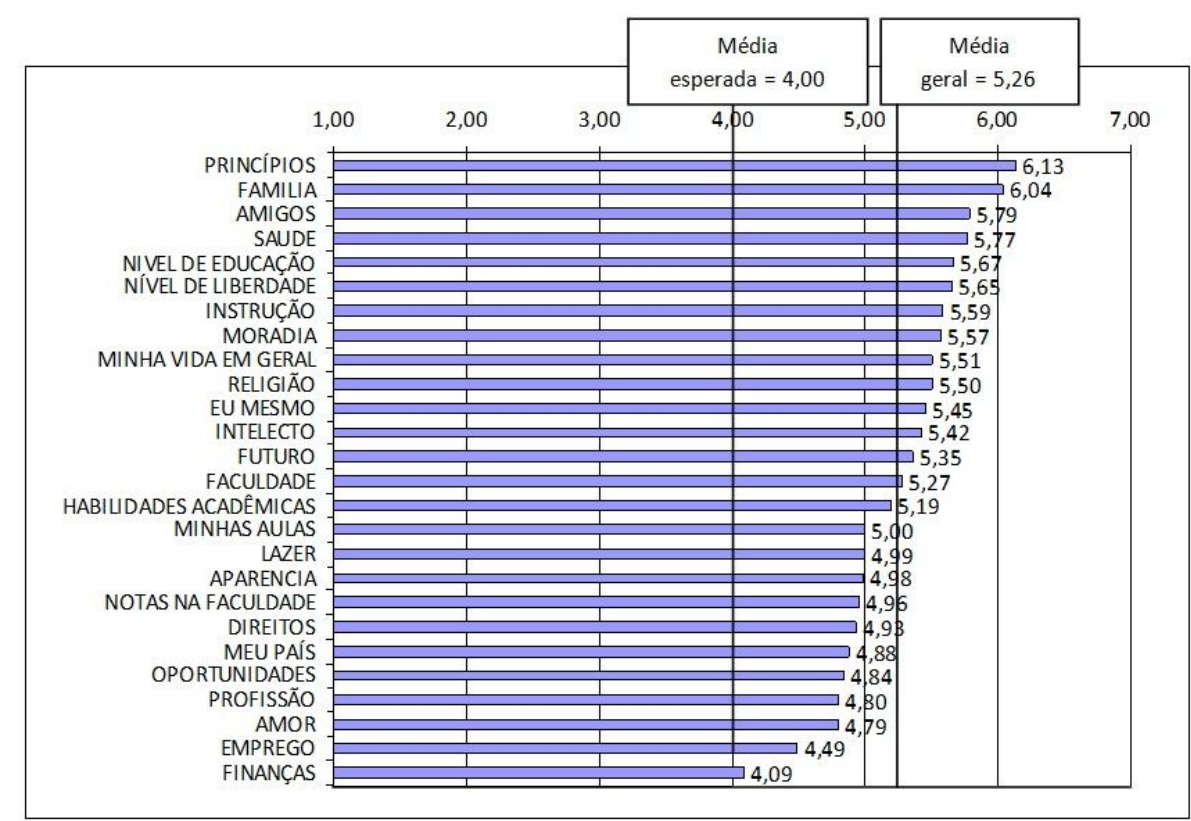

Figura 1. Níveis médios de satisfação com 26 diferentes aspectos da vida ( $\mathrm{N}=388)$

Ao se proceder ao cálculo dos coeficientes de correlação (Bravais-Pearson) entre as principais variáveis investigadas neste estudo, verifica-se que, dos 136 coeficientes calculados (Tabela 2), todos são positivos e 126 são significativos do ponto de vista estatístico.

As diversas medidas dos sentimentos de felicidade mostram-se positiva, significativa e altamente correlacionadas entre si, à satisfação em geral, com os 26 diferentes aspectos da vida do sujeito, com 0 comportamento social, com a percepção do nível socioeconômico e de justiça deste nível, com a avaliação do grau de popularidade e de antissolidão, que por sua vez se correlacionam positivamente entre si de forma importante.

As percepções do nível de riqueza e de justiça deste nível apresentaram coeficientes de correlação positivos e significativos entre si mesmas e com as medidas dos sentimentos de bem-estar subjetivo e de felicidade. 
Tabela 2. Coeficientes de correlação (Bravais-Pearson) entre as principais variáveis constantes deste estudo ( $\mathrm{N}=388$ )

\begin{tabular}{|c|c|c|c|c|c|c|c|c|c|c|c|c|c|c|c|c|}
\hline & 1 & 3 & 4 & 5 & 6 & 7 & 8 & 9 & 10 & 11 & 12 & 13 & 14 & 15 & 16 & 17 \\
\hline 1- FELICIDADE & $0,25^{*}$ & $0,15^{* *}$ & $0,23^{* *}$ & $0,43^{* *}$ & $0,14^{* *}$ & $0,23^{* *}$ & $0,21^{* *}$ & 0,07 & $0,13^{*}$ & $0,13^{*}$ & 0,04 & $0,13^{*}$ & 0,05 & $0,20 * *$ & $0,16^{* *}$ & 0,09 \\
\hline 2 - FELCOM & & $0,58^{* *}$ & $0,93 * *$ & $0,92^{* *}$ & $0,59 * *$ & $0,59 * *$ & $0,47 * *$ & $0,37 * *$ & $0,52 * *$ & $0,26 * *$ & $0,29 * *$ & $0,53^{* *}$ & $0,32 * *$ & $0,60 * *$ & $0,30 * *$ & $0,21 *$ \\
\hline 3- FELTEM & & & $0,84^{* *}$ & $0,81^{* *}$ & $0,44^{* *}$ & $0,52 * *$ & $0,31 * *$ & $0,31 * *$ & $0,35^{* *}$ & $0,28 * *$ & $0,15^{* *}$ & $0,40 * *$ & $0,30 * *$ & $0,40 * *$ & $0,31 * *$ & $0,21 * *$ \\
\hline 4- FELCOMGER & & & & $0,98 * *$ & $0,60 * *$ & $0,63 * *$ & $0,46^{* *}$ & $0,39 * *$ & $0,51^{* *}$ & $0,30 * *$ & $0,26 *$ & $0,53 * *$ & $0,35^{* *}$ & $0,58 * *$ & $0,26^{* *}$ & $0,18^{* *}$ \\
\hline 5- FELITOTAL & & & & & $0,58 * *$ & $0,63 * *$ & $0,47 * *$ & $0,38 * *$ & $0,50 * *$ & $0,31 * *$ & $0,25^{* *}$ & $0,52 * *$ & $0,34^{* *}$ & $0,58 * *$ & $0,28 * *$ & $0,18^{* *}$ \\
\hline 6- SATGERAL & & & & & & $0,62^{* *}$ & $0,38^{* *}$ & $0,21^{* *}$ & $0,24^{* *}$ & $0,27^{* *}$ & $0,13^{* *}$ & $0,30^{* *}$ & $0,25^{* *}$ & $0,36^{* *}$ & $0,40^{* *}$ & $0,24^{* *}$ \\
\hline 7- SATASPEC & & & & & & & $0,42^{* *}$ & $0,28 * *$ & $0,34^{* *}$ & $0,38 * *$ & 0,07 & $0,39 * *$ & $0,23 * *$ & $0,30 *$ & $0,28 * *$ & $0,18^{* *}$ \\
\hline 8- GRAT & & & & & & & & $0,15^{* *}$ & $0,26^{* *}$ & $0,15^{* *}$ & 0,06 & $0,23 * *$ & $0,12^{*}$ & $0,32^{* *}$ & $0,10^{*}$ & $0,20 * *$ \\
\hline 9- SATAMIZ & & & & & & & & & $0,52^{* *}$ & $0,32 * *$ & $0,39 * *$ & $0,84^{* *}$ & $0,32 * *$ & $0,40 * *$ & $0,23^{* *}$ & 0,07 \\
\hline 10- SATINT & & & & & & & & & & $0,24 * *$ & $0,31 * *$ & $0,78^{* *}$ & $0,31 *$ & $0,55^{* *}$ & $0,18^{* *}$ & $0,15^{* *}$ \\
\hline 11- SATFAM & & & & & & & & & & & 0,08 & $0,54^{* *}$ & $0,14^{* *}$ & $0,28 * *$ & $0,10^{*}$ & 0,08 \\
\hline 12- SATSOC & & & & & & & & & & & & $0,60 * *$ & $0,17 * *$ & $0,33^{* *}$ & $0,20 * *$ & $0,13^{*}$ \\
\hline 13- SUPO RSO C & & & & & & & & & & & & & $0,35^{* *}$ & $0,56^{* *}$ & $0,25 * *$ & $0,15^{* *}$ \\
\hline 14- POPUL & & & & & & & & & & & & & & $0,34^{* *}$ & $0,23 * *$ & 0,06 \\
\hline 15- SO LID & & & & & & & & & & & & & & & $0,20 *$ & $0,22^{* *}$ \\
\hline 16- ECONON & & & & & & & & & & & & & & & & $0,33^{* *}$ \\
\hline 17- ECOJUST & & & & & & & & & & & & & & & & \\
\hline
\end{tabular}


Quando se calculou o coeficiente de correlação múltipla, tomando como variáveis independentes os níveis percebidos de popularidade e de antissolidão, os escores nas escalas de gratidão, satisfação geral, satisfação com diferentes aspectos da vida, satisfação com a intimidade das amizades, e como variável dependente uma combinação das escalas para medida da felicidade total (felicidade, felicidade comparativamente com outros e com o tempo) encontrou-se $R=0,79$, coeficiente bastante elevado e altamente significativo do ponto de vista estatístico, explicando $62 \%$ da variância do sentimento de felicidade total.

As relações entre essas variáveis podem ser representadas em um modelo, com os seguintes pesos de cada uma delas na predição do sentimento de felicidade total:

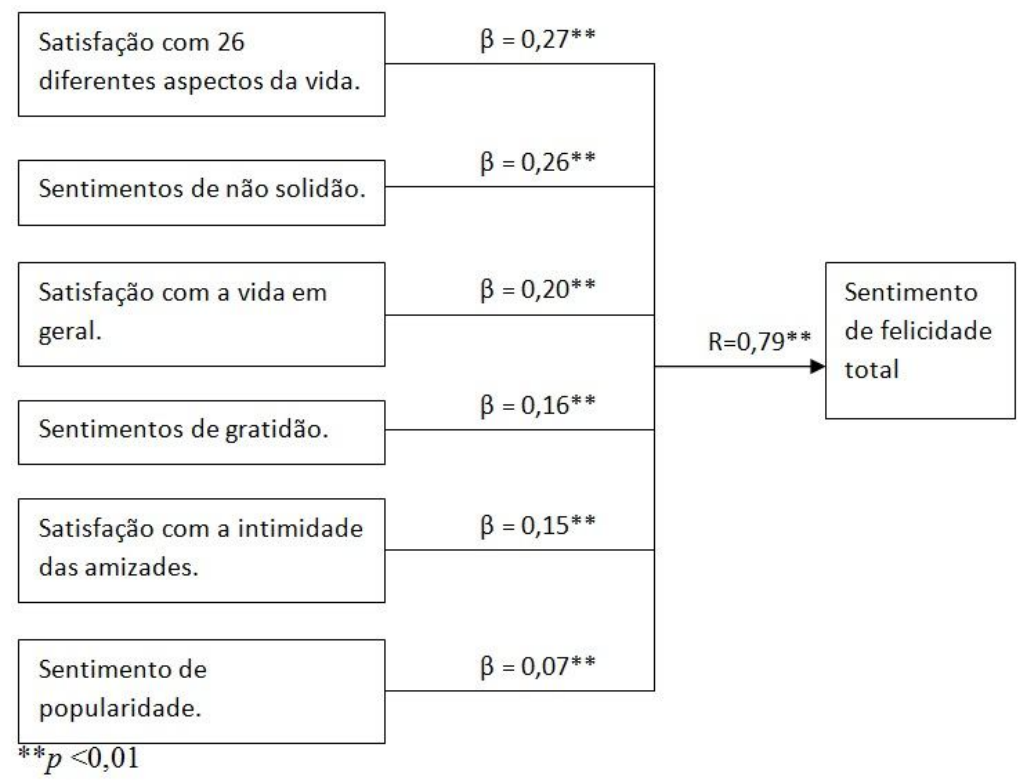

Figura 2. Variáveis preditoras do sentimento de felicidade total e seus respectivos pesos

Ao se comparar os sujeitos divididos em grupos, de acordo com as variáveis biográficas, observa-se que aqueles do sexo masculino apresentam diversos escores médios significativamente diferentes do ponto de vista estatístico $(\mathrm{p}<0,05)$ em relação aos sujeitos do sexo feminino, demonstrando as mulheres menores níveis de solidão, maiores índices de satisfação geral, de gratidão, de sentimentos de felicidade, de felicidade comparativamente aos outros e ao tempo, e de felicidade total.

Outras comparações mostram que os sujeitos com idade mais elevada (acima de 31 anos) apresentam, quando comparados aos sujeitos mais jovens (até 20 anos inclusive), significativamente do ponto de vista estatístico $(p<0,05)$, menor satisfação com suas vidas em geral e, nos 26 diferentes aspectos considerados, menores níveis de felicidade total e mais elevadas posições no continum socioeconômico, acreditando serem mais justas essas posições, quando comparados a seus melhores amigos.

Quando se constituíram grupos de sujeitos que declararam viverem sós, ou que viviam casados ou namorando, os últimos apresentaram-se significativamente $(\mathrm{p}<0,05)$ como mais populares, com menores níveis de solidão, maiores índices de gratidão, de satisfação com diferentes aspectos de suas vidas, experimentando maior felicidade total, declarando encontrarem-se em níveis mais baixos do continuum socioeconômico, na mesma direção dos resultados obtidos por Corbi e Menezes-Filho (2006) e por O liveira e cols. (2009).

Os seguidores e praticantes de uma religião qualquer obtiveram escores significativamente $(p<0,05)$ mais elevados que os não-seguidores e nãopraticantes, mostrando-se mais satisfeitos em geral e com diferentes aspectos de suas vidas, com maiores índices de gratidão, de felicidade total, de felicidade quando comparados a outros ou a outros tempos, de satisfação com aspectos sociais e familiares, e posições mais baixas no contínuo socioeconômico.

Aqueles sujeitos que cursaram escolas privadas de ensino fundamental ou médio, em comparação àqueles que frequentaram escolas no sistema público, apresentam escores significativamente $(p<0,05)$ superiores em diversas variáveis. Assim, consideram-se mais populares, menos solitários, com maior satisfação 
geral e com os diversos aspectos de suas vidas, mais satisfeitos com amizades, com a intimidade dessas amizades, com as atividades sociais das quais participam, com 0 suporte social em geral, demonstrando maiores índices de felicidade total e de gratidão.

Os sujeitos que conhecem outro idioma além do português, em relação àqueles que declararam não conhecer um segundo idioma, mostraram-se significativamente mais populares, experimentando menos estados de solidão. Além disso, estão mais satisfeitos em geral e com diferentes aspectos de suas vidas, com o suporte social, sentem maior felicidade total, situam-se em níveis mais altos no continuo socioeconômico, e acham mais justa essa posição.

Nesse aspecto, ocorre algo muito parecido, e na mesma direção, quando se agrupam os sujeitos que frequentam uma universidade pública ou privada. Os primeiros mostraram-se significativamente mais satisfeitos em geral com suas vidas, experimentam maiores índices de gratidão, avaliam-se em uma posição superior no continuo econômico, e sentem como mais justa essa situação quando comparada à de seu melhor amigo.

Tais diferenças são muito mais claras quando se separam os sujeitos segundo a renda familiar. O s mais abastados, em relação aos de menor nível de renda, de modo consistente com os resultados obtidos por Corbi e Menezes-Filho (2006), sentem-se significativamente $(\mathrm{p}<0,05)$ mais populares, experimentam menor solidão, estão mais satisfeitos em geral e com os diferentes aspectos de suas vidas, experimentam níveis mais elevados de sentimentos de felicidade total, posicionam-se em níveis mais altos no continuo socioeconômico, e percebem como mais justa essa situação.

As mesmas variações são observadas quando se considera a escolaridade do pai e da mãe dos sujeitos. Aqueles cujos pais e/ ou mães são portadores de diplomas de curso superior, comparados aos sujeitos cujos pais não apresentam nível superior de escolaridade, apresentam-se significativamente $(p<0,05)$ como mais satisfeitos em geral e com diversos aspectos de suas vidas, com sentimentos mais positivos em relação ao suporte social, apresentam níveis socioeconômicos mais próximos do ponto extremo "rico" e acreditam mais serem justos esses níveis.

Ao se levar em conta os objetos e bens indicativos do nível socioeconômico do sujeito e/ ou de sua família (tamanho da residência, número de banheiros na casa, número de carros na residência, número de viagens a outros estados ou países, número de empregados domésticos, número de visitas a museus, número de vezes em que se hospedou em hotéis no último ano, número de livros existentes em casa, número de vezes que jantou fora de casa e gasto médio com esses jantares), verifica-se que os sujeitos pertencentes ao grupo com maiores e melhores indicadores apresentam, significativamente $(\mathrm{p}<0,05)$, em comparação aos sujeitos do grupo com menores níveis sócio-econômicos, maior popularidade, menor solidão, maior satisfação em geral e com diferentes aspectos de suas vidas, maiores índices de gratidão, mais sentimentos positivos para com o suporte social, maiores sentimentos de felicidade total.

Por último, e como um resumo de todas as diferenças relatadas neste estudo, relacionadas às características socioeconômicas dos sujeitos, quando se formaram grupos extremos nas variáveis percepção do posicionamento no continuo socioeconômico, e justiça dessa posição em relação ao seu melhor amigo, os resultados mostram diferenças estatisticamente significativas $(\mathrm{p}<0,05)$. O s dados indicam que o grupo de sujeitos que se posiciona de forma mais positiva no continuo socioeconômico, e que sente como mais justa essa posição, apresenta-se com escores mais elevados em popularidade, menores índices de solidão, maior satisfação em geral com suas vidas e com aspectos particulares da mesma, maiores índices de gratidão, de satisfação com o suporte social e maiores níveis de felicidade total.

\section{Discussão e considerações finais}

Em que pesem as limitações deste estudo, relativas, por exemplo, ao processo de amostragem dos sujeitos, à inclusão de outras categorias de indivíduos que não somente estudantes universitários, ao desenvolvimento de novas formas de mensuração dos construtos aqui examinados, ao aprimoramento das escalas utilizadas, diversas contribuições importantes puderam ser oferecidas.

O s resultados expostos e analisados anteriormente oferecem novos dados para 0 estudo da qualidade das escalas, sobretudo aquelas destinadas à medida dos diferentes aspectos da felicidade, satisfação com a vida e com seus diferentes aspectos, sentimentos de gratidão, satisfação com o suporte social, em suas diferentes nuances. Tais dados nos oferecem importante patamar, a partir do qual podem e devem ser desenvolvidos e aprimorados instrumentos capazes de mensurar as variáveis em pauta, e que estariam certamente presentes em qualquer iniciativa futura de obtenção de índices nacionais indicativos dos níveis de felicidade, de bem-estar subjetivo e de satisfação 
experimentados pelo povo brasileiro. Esse fato poderia atender ao preconizado por Diener (2000) e pelo prêmio Nobel de economia de 2002, Daniel Kahneman, que defendem a necessidade de estudos capazes de determinar índices nacionais de sentimento de bem-estar subjetivo ou de felicidade, o que de certa forma já havia sido sugerido anteriormente pelo também prêmio Nobel de economia Joseph Stiglitz, ao defender que os países necessitariam diminuir a importância conferida aos números da indústria e comércio e enfatizar os efeitos desses números nas pessoas e na sociedade como um todo.

Um elemento recorrente e presente nos dados expostos anteriormente refere-se aos escores médios apresentados pelos sujeitos deste estudo, que demonstram altos e positivos sentimentos de felicidade, satisfação com a vida em geral e com seus diferentes aspectos, e gratidão, tal como já demonstrados em estudos anteriores com estudantes e professores universitánios (D ela Coleta \& D ela Coleta, 2006, 2007) e que parecem se constituir em uma constante entre os sujeitos brasileiros de diferentes grupos, conforme demonstrado por diversos pesquisadores estrangeiros citados anteriormente.

0 exame dos coeficientes de correlação entre as variáveis cobertas por este estudo mostra que as diferentes medidas de felicidade correlacionam-se positiva e significativamente entre si, com a satisfação com a vida e com seus diferentes aspectos, com os sentimentos de gratidão, com a satisfação com a vida social, com os sentimentos de popularidade e de não solidão experimentados pelos sujeitos, com as diferentes medidas do nível socioeconômico do sujeito e com a percepção de justiça do mesmo.

Importante ressaltar aqui que a satisfação com a vida em geral e com seus diferentes aspectos, os sentimentos de popularidade, não-solidão, gratidão e a satisfação com os aspectos relativos à intimidade das amizades guardam correlação múltipla de $R=0,79$ com o sentimento de felicidade total. Esses resultados mostram que o sentimento de felicidade de um indivíduo pode, em grande parte, ser predito pela satisfação com sua vida, com suas amizades, com sua popularidade e com os sentimentos de gratidão experimentados. $\mathrm{O}$ que chama a atenção nesses dados é a magnitude do coeficiente de correlação múltipla explicando nada menos que $62 \%$ da variância dos índices de felicidade total, sem dúvida elevada e consistente, o que permite segurança na interpretação dessas relações.

Outro aspecto das análises efetuadas e descritas na seção anterior, que apresentou uma enorme quantidade de informações, diz respeito às diferenças entre as médias nas diversas variáveis consideradas quando se leva em conta as características biográficas dos sujeitos. Assim, foram encontradas diferenças estatisticamente significativas entre os grupos ao se considerar o sexo, a religião, o conhecimento de outro idioma, a frequência a escolas e universidades públicas vesus privadas, a renda familiar, o nível de escolaridade dos pais, a idade, 0 estado civil e indicadores objetivos do nível socioeconômico do sujeito, complementando ou confirmando os resultados obtidos, entre outros, por Rodrigues e Silva (2010), Oliveira e cols. (2009), Corbi e Menezes-Filho (2006).

Chama a atenção, quando comparado à literatura revisada, o fato de que os sujeitos do grupo feminino, casados ou namorando, praticantes de uma religião, que frequentam escolas privadas no ensino fundamental e pública no ensino superior, que declaram conhecer outro idioma além do português, com renda mais alta, com idade mais baixa (até 20 anos inclusive), com posse de objetos e bens indicativos de nível socioeconômico mais elevado, e alto nível educacional dos pais apresentam significativamente níveis mais elevados de satisfação com a vida em geral e com seus diferentes aspectos, e nas diversas medidas do sentimento de felicidade.

Esses dados parecem dizer, de forma inequívoca, que uma série de variáveis demográficas, como a juventude, o sexo feminino, a convivência sistemática com outro, a prática de uma religião, maiores níveis de educação e, sobretudo, mais elevados níveis sócioeconômicos facilitam ou mesmo determinam maiores índices de satisfação, bem-estar subjetivo e felicidade, como já identificado em estudos anteriores (Corbi \& Menezes-Filho, 2006; O liveira \& cols., 2009; Rodrigues \& Silva, 2010).

Os achados deste e de outros estudos conduzidos no Brasil apresentam enorme quantidade de informações sobre o comportamento das variáveis associadas à felicidade e ao bem-estar subjetivo, e suas relações com características demográficas dos sujeitos. Por outro lado, demonstram a necessidade de que estudos futuros possam privilegiar a coleta de dados junto a sujeitos não estudantes, cobrindo outras amostras ainda não examinadas, visando responder a uma das lacunas deste e de outros estudos. Ao mesmo tempo, há necessidade de futuros estudos destinados a ampliar a cobertura, confiabilidade e validade das escalas para medida das diversas variáveis associadas à felicidade e ao bem-estar subjetivo.

Essas sugestões de novos estudos tornariam possível complementar uma série de limitações identificadas neste estudo envolvendo, entre outros, a amostra composta unicamente por universitários que 
tiveram acesso à internet para responder aos instrumentos, a utilização de um conjunto de escalas já empregadas em estudos anteriores, que certamente não conseguem representar a totalidade das dimensões dos construtos, a composição dos grupos de sujeitos em função de suas características biográficas somente após 0 recolhimento dos dados, 0 que implica a impossibilidade de estudar algumas combinações dessas variáveis.

O s estudos já realizados, este agora apresentado e outros futuros, permitirão oferecer as bases metodológicas capazes de sustentar a realização de levantamentos decenais, ou talvez até anuais, para a determinação de um índice nacional de felicidade, 0 que poderá fornecer subsídios para definir a situação em que se encontra a população ou amostras de brasileiros nos diferentes aspectos cobertos pela possível futura nova inclusão da busca da felicidade como um direito básico do ser humano, ao lado de educação, saúde, moradia, emprego, como previsto no artigo 6음 da Constituição Brasileira.

\section{Referências}

Albuquerque, A., \& Tróccoli, B. T. (2004). Desenvolvimento de uma escala de bem-estar subjetivo. Psicdoja, Teeria e Pequisa, 20(2), 153164.

Albuquerque, F. J. B., Noriega, J. A. V., Coelho, J. A. P. M., Neves, M. T. S., \& Martins, C. R. (2006). Valores humanos básicos como preditores do bem-estar subjetivo. Psica, 37(2), 131-137.

Albuquerque, F. J. B., Noriega, J. A. V., Martins, C. R., $\&$ Neves, M. T. S. (2008). Locus de controle e bem-estar subjetivo em estudantes universitários da Parába. Psicología para América Latina, 13. Disponível:

<http:/ / pepsic.bvsalud.org/ scielo.php?script=sci arttext\&pid=S1870-

350X 2008000200011\&lng=pt\&nrm=iso>.

Recuperado: 11 dez. 2011.

Chaves, S. S. S., \& Fonseca, P. N. (2006). Trabalho docente: que aspectos sociodemográficos e ocupacionais predizem o bem-estar subjetivo? Psio, 37(1), 75-81.

Corbi, R. B., \& Menezes-Filho, N. A. (2006). Os determinantes empíricos da felicidade no Brasil. Revista deEconamia Pdítica, 26(4), 518-536.

Costa, L. S. M., \& Pereira, C. A. A. (2007). Bem-estar subjetivo: aspectos conceituais. Arquivos Brasileros dePsicoøa, 59(1), 72-80.
Dela Coleta, J. A., \& D ela Coleta, M. F. (1997). Satisfação com aspectos da vida: uma comparação entre 41 culturas. [Resumo]. Em Sociedade Interamericana de Psicologia (O rg.), Resumos XXVI Congesso Interamericamo dePsicdoga (pp. 138139). São Paulo: SIP.

Dela Coleta, J. A., \& D ela Coleta, M. F. (2006). Felicidade, bem-estar subjetivo e comportamento acadêmico de estudantes universitários. Psicdoga emEstudo, 11(3), 533-539.

Dela Coleta, J. A., \& D ela Coleta, M. F. (2007). Felicidade e bem-estar subjetivo entre professores universitários. Quaestio(UNISO), 9(2), 131-143.

Dela Coleta, J. A., Dela Coleta, M. F., \& Diener, E. (1996). Estudo transcultural do sentimento de satisfação e de felicidade [Resumo]. Em Sociedade Brasileira de Psicologia (O rg.), Resumos de Comunicacões Científicas XXVI Remião Anual de Psicdoga (p. 184). Ribeirão Preto: SBP.

Diener, E. (2000). Subjective well-being: the science of happiness and a proposal for a national Index. American Psychdogst, 55(1), 34-43.

Diener, E. (Ed.). (2009). The Saience of well-being the clleted works of EdDiene. Nova Iorque: Springer.

Diener, E., Diener, M., \& Diener, C. (1995). Factors predicting the subjective well-being of nations. Jaumal of Pesconality and Social Psydhogy, 69, 851864.

Diener, E., \& Oishi, S. (2000). Money and happiness: income and subjective well-being across nations. Em E. Diener \& E. M. Suh (Eds.), Culture and subjetive well-being (pp.185-218). Cambridge: The MIT Press.

Diener, E., \& Suh, E. M. (Eds.). (2000). Culture and subjetivewdl-being Cambridge, MA: MIT.

Diener, E., Suh, E. M., Lucas, R. E., \& Smith, H. L. (1999). Subjective well-being: three decades of progress. Psydhdogial Bullein, 125(2), 276-302.

Ferraz, R. B., Tavares, H., \& Zilberman, M. L. (2007). Felicidade: uma revisão. Revista dePsialoga Clínica, 34(5), 234-242.

Giacomoni, C. H. (2004). Bem-estar subjetivo: em busca de qualidade de vida. Temas em Psicdoga da SBP, 12(1), 43-50.

Gonçalves, D. M., \& Kapczinski, F. (2008). Transtorno mental, indicadores demográficos e satisfação com a vida. Revista deSaúdePública, 42(6), 1060-1066. 
Guedea, M. T. D., Albuquerque, F. J. B., Tróccoli, B. T., Noriega, J. A. V., Seabra, M. A. B., \& Guedea, R. L. D. (2006). Relação do bem-estar subjetivo, estratégias de enfrentamento e apoio social em idosos. Psiølogia RelexãoeCńtica, 19(2), 301-308.

Inglehart, R., \& Klingemann, H. D. (2000). Genes, culture, democracy and happiness. Em E. Diener \& E. M. Suh (Eds.), Cultureand subjetive wdl-bing (pp. 165-183). Cambridge: MIT.

Linley, A., Joseph, S., Harrington, S., \& Wood, A. M. (2006). Positive psychology: past, present and (possible) future. The Jamal of Positive Psychdogy, 1(1), 3-16.

Lucas, R. E., \& Gohm, C. L. (2000). Age and sex differences in subjective well-being across cultures. Em E. Diener \& E. M. Suh (Eds.), Culture and subjetive well-bing (pp. 291-317). Cambridge: The MIT Press.

Oliveira, G. F., Barbosa, G. A., Souza, L. E. C., Costa, C. L., Araújo, R. C. R., \& Gouveia, V. V. (2009). Satisfação com a vida entre profissionais de saúde: correlatos demográficos e laborais. Revista Biótica, 17(2), 319-334.

Otta, E., \& Fiquer, J. T. (2004). Bem-estar subjetivo e regulação de emoções. Psicogáa em Reista, 10(15), 144-149.

Passareli, P. M., \& Silva, J. A. (2007). Psicologia positiva e 0 estudo do bem-estar subjetivo. EstudosdePsicdoja, 24(4), 513-517.

Ribeiro, J. L. P. (1999). Escala de Satisfação com 0 Suporte Social (ESSS). Análise Psicoóġa, 3(XVII), 547-558.

Sobre os autores:

José Augusto Dela Coleta é doutor em Psicologia pela Fundação Getúlio Vargas, Psicólogo pela USP-Ribeirão Preto, professor titular aposentado da Universidade Federal de Uberlândia, professor de cursos de pós-graduação e consultor de empresas.

José E duardo Ferreira Lopes é doutorando em Administração na FEA-USP/ Ribeirão Preto, Administrador pela Universidade Federal de Uberlândia, Bacharel em Ciências da Computação pela Universidade Federal de Viçosa e professor da Universidade Federal de Uberlândia.

Marilia Ferreira Dela Coleta é doutora em Psicologia pela Universidade de Brasília, Psicóloga pela Universidade Federal Fluminense, professora do Instituto de Psicologia da Universidade Federal de Uberlândia.

Contato com os autores:

Rua Seriemas, 455, Cidade Jardim - CEP 38412-158. Uberlândia, MG. Email: dela.coleta@ netsite.com.br 